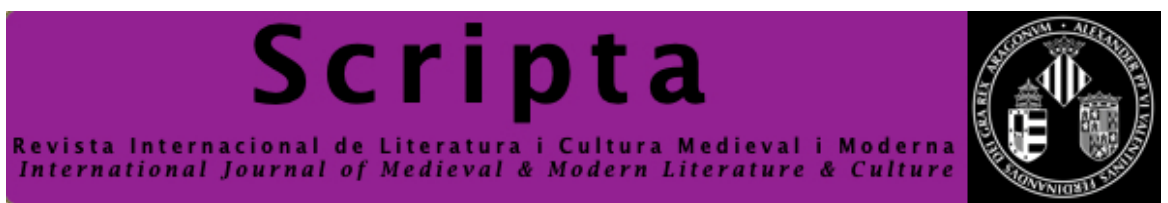

\title{
Il transfert di Curial
}

\author{
The transfert of Curial
}

\author{
Anna Maria Compagna \\ compagna@unina.it \\ Università di Napoli Federico II \\ A SALVATORE CERASUOLO
}

\begin{abstract}
Resum: I risvolti sovranazionali riscontrati nei tre protagonisti dei romanzi del Quattrocento catalano (Jacob Xalabín, Curial e Tirant) consentono di procedere a un'analisi del Curial e Güelfa che ne sottolinea l'identità multipla e l'apertura culturale del processo di formazione del protagonista, nella sua ascesa economica e sociale. L'evoluzione del protagonista, grazie a un meccanismo psicologico, oggi riconosciuto come tale, consente il lieto fine del romanzo, diversamente da quanto avviene negli altri due, dove i protagonisti, Jacob e Tirant, rimangono sempre gli stessi. Grazie allo spostamento, al transfert, Curial non rimuove quello che lo minaccia, ma riesce a superarlo, se riguarda un altro individuo, e questo permette il suo successo finale, senza essere vittima d'impulsi interni inaccettabili.
\end{abstract}

Paraules clau: Identità/alterità, multiplo/singolo, apertura/chiusura culturale, onore/disonore, ascesa/ discesa economica e sociale, rimozione/spostamento.

Abstract: The supranational implications found in the three protagonists of the novels in the "Catalan quattrocento" (Jacob Xalabín, Curial and Tirant) allow an analysis of Curial e Güelfa that underlines the multiple identity and cultural openness of the protagonist's training process, in his economic and social rise. The evolution of the protagonist, thank to a psychological mechanism, now recognized as such, allows the happy ending of the novel, unlike what happens in the other two, where the protagonists, Jacob and Tirante, always remain the same. Thanks to the movement, to the transfert, Curial does not eliminate what threatens it, but manages to overcome it, if it is another individual, and this allows its final success, without being the victim of unacceptable internal impulses.

Keywords: Identity/alterity, multiple/single, openness/cultural closure, honor/dishonor, economic and social promotion /decline, removal/displacement

\footnotetext{
* Si tratta della revisione di quanto ho detto al Convegno Internazionale «Curial e Güelfa» La cavalleria umanistica italiana del XV secolo. (Università di Verona, 10-12 ottobre 2016), nella comunicazione dal titolo Identità multipla e apertura culturale nel 'Curial e Güelfa'. Gli altri interventi presentati appariranno in un volume di saggi di prossima pubblicazione.
} 
Dopo che gli studi di Abel Soler hanno identificato in maniera plausibile e convincente, se non addirittura definitiva, l'ubicazione napoletana e l'autoria di Enyego d'Àvalos, plausibile e convincente (Soler 2017a e 2017b), sembrerebbe inutile tornare sulla dimensione internazionale della narrativa catalana nel secolo XV e sulle forme e sui contenuti che rispecchiano il carattere sovranazionale della Corona d'Aragona (Ferrando 1997, Compagna 2008-2009, Ferrando 2012). ${ }^{1}$

Eppure, in quest'ottica, il contesto che fin dall'inizio propone il Curial e Güelfa continua a essere particolarmente interessante: si tratta di un contesto italiano, sia dal punto di vista della forma (la relazione con Boccaccio) che da quello del contenuto (la storia di un giovane accolto alla corte del marchese del Monferrato). Certo sembrerebbe un contesto più angioino che aragonese, ma la storia dell'arte ci ha insegnato che fra le due etichette non è facile distinguere (del resto i matrimoni fra le due dinastie sono continui, quasi quanto le guerre...).

Via via che si entra nel vivo del Curial il contesto italiano si dilata e ne varca confini geografici, si internazionalizza, anche se la relazione del romanzo con l'Italia continua a essere prioritaria, così come pure il suo legame con la letteratura italiana e umanista, che già aveva fatto sentire i suoi influssi sulle lettere catalane, prima ancora che in quelle castigliane.

Però Curial è un romanzo cavalleresco complicato, alla cui identità mi pare che si addica la definizione di multipla (italiana, catalana, angioina, aragonese, medievale, umanista...), proprio per l'apertura culturale che documenta. Apertura verso la tradizione catalana, all'interno della quale si inserisce, quindi verso quello che viene prima, nei generi letterari diversi che vanno assumendo una loro fisinomia specifica, (storiografia, romanzo, lirica trobadorica), ma anche apertura verso quello che lo circonda nel presente (ancora romanzo, lirica...). I rapporti paradigmatici che l'opera instaura con il canone, all'interno del quale trova il suo spazio, non sono meno intensi di quelli, che potremmo definire sintagmatici, che affiorano e che innovano la tradizione.

Un indice dei luoghi entro cui l'azione si svolge consente di affermare che la cornice geografica è assolutamente reale, seguiamo Curial che si muove fra città a paesi, anche distanti fra loro, percorre, chilometri e miglia per terra e per mare: dal Monferrato, in Italia, alla Germania, dall'Austria a Parigi, dalla Grecia alla Terra Santa e a Tunisi...

1 Badia, Torró 2015 si riferivano a concetti analoghi, dopo aver parlato semplicemente di corts ibèriques. nella loro edizione del 2011: già Butinya, Gros (2013: 523) avevavo notato la disponibilità di Badia, Torró 2011 a rivedere determinate posizioni: «l'absència d'emmarcament del Curial en l'Humanisme per part de Badia i de Torró, s’hi compensa amb el seu reconeixement del fort influx boccaccià». Ma quale era la conoscenza del mondo classico greco in Spagna all'epoca di questo romanzo? Non si aveva forse più familiarità con quella latina o con la Bibbia? Comunque, se per transfertt intendiamo in senso più lato un processo di identificazione che permette una catarsi, credo che questo sia un fenomeno abbastanza diffuso.

Tutto sommato nei miti di Bellerofonte e in quello di pereseo troviao qualcosa di simile, anche se entrambi gli eroi superano una serie di prove in terre lontane con aiuto divino e senza che siano presenti «altri» : essi non hanno alcuna colpa e caso mai, come Bellerofonte sono stati accusati ingiustamente (cfr.Giuseppe e la moglie di Putifar in Genesi). Laio, padre di Edipo, invece è il primo uomo ad esercitare l'amore pederastico, con Crisippo, figlio del re che lo ospita: non so se il divieto di avere figli possa esser messo in relazione con questa colpa, almeno i testi non lo dicono. Non riesco ad andare oltre; mi domando se in Ovidio, invece non ci sia qualcosa.... 
Non altrettanto si può dire se osserviamo quello che c'è all'interno della cornice. Quando si svolge la storia narrata? Chi sono i personaggi coinvolti, storici e immaginari? Riassumiamo quello che viene raccontato:

Molto tempo fa, dice l'anonimo... Quindi siamo in anni non definiti.

Neanche il luogo viene precisato all'inizio: il manoscritto presenta uno spazio lasciato in bianco, proprio dove ci aspetteremmo questa informazione..., ingenuamente riempito in un secondo momento da un'altra mano, nel tentativo di circostanziare la vicenda.

Poi l'anonimo fa riferimento a quello che ha letto (letto non sentito), a proposito di un gentiluomo chiamato...

Neanche questo nome ci viene detto, perché c'è un altro spazio bianco nel manoscritto (che questa volta viene rispettato).

Comunque leggiamo che il gentiluomo senza nome, al quale si fa riferimento, è più saggio e cortese che ricco, visto che possiede solo un'umile casa. Con la moglie bellissima, chiamata Onorata, egli vive poveramente e onestamente. I due fanno di tutto per acquistare la grazia di Dio, senza obblighi mondani. E così in vecchiaia sono premiati e hanno un figlio, che chiamano Curial, una creatura più bella delle altre.

L'esordio non sembra molto diverso da quello del Blaquerna, che a sua volta rimandava al modello agiografico...

Presto il padre muore e la madre non permette a Curial di allontanarsi (sembra la mamma di Perceval). Allora il ragazzo scappa dalla vita povera che gli tocca e va dal giovane marchese di Monferrato (finalmente un riferimento geografico preciso), che da poco aveva ereditato il marchesato del padre: quindi è orfano come Curial, ma è ricco e ha un marchesato. Ha anche una sorella minore chiamata Guelfa.

Questi primi segmenti narrativi sono preceduti da un breve esordio, (Quanto è grande il pericolo, quanti sono gli affanni e le angosce di coloro che soffrono le pene d'amore!), dove l'anonimo motiva la sua narrazione: se il caso che narra sarà giudicato con assennatezza, molti dovrebbero guardarsi dall'introdursi in un simile amoroso, ma doloroso cammino, anche se i due protagonisti, dopo lungo tempo, ottengono il premio dei loro affanni. Ci troviamo, quindi davanti a una storia d'amore, un caso particolare, come direbbe anche Boccaccio, che prende le mosse da un modello che non è molto distante da quello agiografico/ biografico, anche se più che la bontà del protagonista, sembra contare la sua bellezza.

Di chi sei? chiede il marchese a Curial, quando lo ha davanti a sé. Signore sono vostro, risponde il ragazzo. E per la sua bellezza al marchese piace che lui sia suo e lo dice ai suoi. Finalmente il marchese chiede il suo nome (è più importante di chi si è che chi si è) e lo fa suo cameriere personale. Curial alla bellezza unisce la grazia, entrambi doni di Dio, ma per le altre doti che deve 
avere un cavaliere è necessaria la sua formazione.

Anche Guelfa è bella: a tredici anni aveva sposato il duca di Milano, che dopo due anni era morto lasciandola erede del ducato.

La sorella del duca di Milano, Andrea, a sua volta aveva sposato il marchese che ne era talmente preso da mettere un po' da parte Curial, lasciando che possa approfittarne per studiare grammatica, logica, retorica, filosofia e diventare anche poeta famoso (c'è un nesso fra filosofia e poesia?² Ausiàs March sarà presentato come poeta e filosofo nel frontespizio dell'editio princeps del 1539). Abbiamo quindi una coppia di coppie che s'incrocia con una coppia di fratello e sorella, il marchese di Monferrato e Andrea, sorella del duca di Milano, Curial e Guelfa, sorella del marchese di Monferrato, da una parte, e un fratello e sorella, il marchese di Monferrato e Guelfa, e il duca di Milano e Andrea. Quindi Curial ha una sorta di doppio nel Marchese e Guelfa in Andrea.

Pare che a parte Curial solo le donne abbiano un nome, la madre Onorata, la sorella del marchese Guelfa, la sorella del duca di Milano Andrea.

Guelfa, vedova, torna a vivere nel castello del marchese e visto che questi non si preoccupa di darle un nuovo marito, non potendo resistere agli appetiti della carne (si respira l'aria delle novelle di Boccaccio e si è parlato della prima novella della quarta giornata, ma l'annunciato lieto fine del Curial fa pensare che il nostro autore voglia prendere le distanze dal tragico epigono della novella), decide di amare segretamente un giovane e si innamora di Curial, procurandone l'ascesa, attraverso gioie e denari che gli consentono di diventare cavaliere. Egli accetta la protezione e consegue tutto quello che si addice a un nobile giovane e valoroso.

Melchiore Pandone, amministratore di Guelfa, dopo avere esaltato le virtù di Curial davanti a Guelfa, si fa carico di educarlo e formarlo secondo i canoni della incarica della cortesia.

A questo punto inizia l'azione.

La mette in moto l'invidia di due anziani cavalieri, Ansaldo e Ambrosio, che sorprendono Curial e Guelfa mentre si baciano e lo dicono al marchese, che accusa il giovane. È evidente il dejà vu in tutto questo. Curial si difende e si dice disposto a combattere contro chi lo accusa. Il marchese gli crede, ma comunque Curial, per ubbidire al marchese, vuole evitare di continuare a vedere Guelfa e perciò parte alla ventura dopo essersi accomiatato dalla vedova del duca di Milano... Il tutto sembra abbastanza stereotipato e noto... La situazione non sembra molto lontana da quella di Tristano, soltanto che, al posto della moglie del signore, c'è la sorella...

Quello, però, che qui tengo a sottolineare è che Curial, che non aveva combattuto contro i due che lo avevano accusato, combatterà contro due accusatori di adulterio della duchessa d'Austria, cugina della regina di Ungheria, Cloto, operando quello che oggi in psicologia si chiama spostamento,

2 Mi pare evidente anche qui l'impronta umanistica che l'autore imprime attraverso il recupero della tradizione classica. 
transfert, cioè un processo automatico e inconscio, attraverso il quale una emozione interna, derivante da un impulso inaccettabile, viene spostata su un oggetto sostitutivo. ${ }^{3}$ Del resto, Guelfa gli aveva detto, al momento della sua partenza che, se lui avesse vinto gli avversare che avrebbe incontrato nel suo vagare, da allora in avanti non ci sarebbe più stato nessuno avrebbe osato parlare di loro, perché avrebbero avuto paura di lui: chi avrebbe difeso l'onore altrui, con ancora maggiore coraggio avrebbe difeso il suo.

Il gioco degli specchi, già abbozzato nell'incontro di Curial, giovane povero, privo di possedimenti, orfano, con il marchese, giovane ricco con un marchesato, orfano, si ripete e si conferma come procedimento narrativo.

Il cavaliere Jacob de Cléves diventa un alter ego di Curial. Già il marchese era stato introdotto con alcuni caratteri uguali a quelli di Curial: giovane, orfano, ma ricco, con addirittura un marchesato. Anche Jacob entra in scena con qualcosa in comune con Curial: è accusato, ma di qualcosa che è andato ben oltre quello di cui era stato accusato Curial: Jacob de Cléves deve difendere la duchessa d'Austria accusata di adulterio, Curial, nel caso, avrebbe dovuto difendere la sorella, non la moglie, del marchese di Monferrato di che cosa? Di un bacio? Il bacio rappresenta l'incontro carnale?

Anche in questo caso gli accusatori della duchessa d'Austria sono due e c'è un solo difensore. Perciò Curial si proporrà al fianco del suo alter ego nell'impresa e Del resto è più onorevole difendere la causa altrui che non la propria.

\footnotetext{
3 I riferimenti bibliografici possibili possono partire per ora solo da indagini di tipo psicologico su personaggi teatrali, quindi da studi che si affidano a un'altra costruzione testuale. Si pensi ai lavori di Francesco Orlando su Fedra e Misantropo, ad esempio, o le Scene perturbanti e rimosse di un libro di Alonge, o La stanza della tortura del Pirandello di Macchia. Certo siamo lontani dall'area cronologica, ma questo tipo di indagini possono comunque trasmettere qualche utile suggestione, per smuovere le acque fra tanti «agenti dell'Impero». Certo qui si tratta senz'altro della riscrittura di una leggenda di tradizione germanica (Compagna 1995 è Soler 2017b: 861 e sgg. sono solo due dei possibili riferimenti bibliografici), nella quale affiora probabilmente il motivo della falsa accusa ben noto al folclore (Thompson 1967: 295), e non credo che si possa trovare un qualche riferimento attinente nella mitologia classica (quello delle armi di Achille indossate da Patroclo non mi sembra proponibile). Comunque, le prime due imprese del nostro eroe mi sembrano rientrare nel tipo di «rituale» iniziatico, con il tipico vagare in terre straniere, ecc., tutti temi molto comuni, anche se mi sembra che nessuno ripropone in pieno il nostro schema. Tuttavia, riflettendoci, il mito ed il suo valore come «modello» in cui identificarsi, forse si possono definire transfert. Si veda il lavoro di Cambi 2000. Resta il dubbio su quale potesse essere la conoscenza del mondo classico greco in Spagna all'epoca del tuo romanzo. Non si aveva forse più familiarità con quello latino e con la Bibbia? Comunque, se per transfert intendiamo in senso più lato un processo d'dentificazione che permette una catarsi, credo che questo sia un fenomeno abbastanza diffuso. Tutto sommato i miti di Bellerofonte o anche quello di Perseo hanno molto in comune con il nostro, anche se entrambi gli eroi superano una serie di prove in terre lontane con aiuto divino e senza che siano presenti «altri «: essi non sono veramente colpevoli e caso mai, come Bellerofonte sono stati accusati ingiustamente (cfr. Giuseppe e la moglie di Putifar nella Genesi). Laio, padre di Edipo, invece è il primo uomo ad esercitare l'amore pederastico, con Crisippo, figlio del re che lo ospita: non so se il divieto di avere figli possa esser messo in relazione con questa colpa, almeno i testi non lo dicono. Non è facile andare oltre; ma c’è da chiedersi se in Ovidio, invece non ci sia qualcosa.... Ringrazio Luisa Breglia degli stimolanti suggerimenti che mi ha fornito.
} 
Quando Curial si era accomiatato da Guelfa, questa gli aveva ricordato che era suo e che l'unica cosa che lei desiderava era la crescita del suo onore, che poteva avvenire solo attraverso le armi. La scena si era chiusa con il venire meno della giovane e l'autore del romanzo aveva detto di tralasciare i dettagli della tristezza dei due innamorati per brevità, raccontando solo quello che gli sembrava necessario al conforto e al piacere di quelli al quale si rivolgeva. Conforto e piacere dalla tristezza? O meglio, conforto e piacere per il destinatario, per il pubblico, evitando d'insistere troppo sulla tristezza attraverso la quale sarebbero passati i protagonisti del romanzo. Conforto e piacere che forse il modello proposto, l'ascesa economica e sociale del protagonista, avrebbe potuto dare...

Curial, durante il suo cammino, prima di avere sconfiggere con Jacob de Clèves gli accusatori della duchessa d'Austria, era arrivato in una città ungherese (il che non quadra, perché dal Monferrato non si passa per l'Ungheria, andando in Austria), e aveva affrontato il cavaliere Harrich de Fontaynes, in difesa di Auger Bellian, un vecchio cavaliere accusato di omicidio e vince il suo avversario, che i figli del vecchio non hanno il coraggio di affrontare.

Già qui c'era stato uno spostamento, un transfert (traslazione): Curial aveva difeso una causa altrui, invece della propria, e questo ne aveva determinato la crescita dell'onore... Poi nell'episodio successivo, quello con la duchessa d'Austria accusata di adulterio, il transfert si fa ancora più netto, l'onore di Jacob de Clèves non cresce quanto quello Di Curial, ma partecipa alla crescita di quello di Curial: nel momento in cui Jacob cerca di dissuadere Curial dal farsi i fatti degli altri: secondo Jacob, badando ai fatti propri, si fa già abbastanza ( $\mathrm{ma}$ in realtà egli ha paura di perdere l'aiuto promesso da Curial, al punto che quando non riesce a dissuaderlo dal duello, cerca di dissuadere l'avversario). Così il secondo alter ego di Curial finisce di esserlo.

Del resto già il primo alter ego di Curial, il marchese, si era differenziato da lui per la sua ricchezza e potere... L'identità di Curial si costruisce attraverso la sua differenziazione dagli altri: giovane e orfano come il marchese, ma non ricco e potente come lui; accusato come Jacob, ma pronto a difendere l'onore altrui, diversamente da Clèves!

L'identità si trova anche attraverso l'alterità. Si procede per sinonimi (differenziazione, alterità) e contrari (identità/alterità, multiplo e complesso/singolo e semplice).

L'identità di Curial si definisce a poco a poco, differenziandosi dagli altri.

E la dimensione italiana dell'esordio ampia via via i suoi confini: quando Curial arriva dall'imperatore, si dice che egli aveva la fama di essere il più bello e migliore uomo d'arme del mondo e che i molti signori che vengono per vedere la battaglia erano della Germania, della Francia, dell'Italia e di tanti altri luoghi.

È a questo punto che Curial chiede all'imperatore di farlo cavaliere. E così, dopo la sua vittoria l'imperatore invoca l'onore e la gloria di tutta la cavalleria del mondo, all'interno della quale colloca già Curial: all'imperatore piacerebbe addirittura essere come lui e che lui fosse imperatore. 
Eppure Curial aborrisce dal procurare la morte a un qualche cavaliere: «non piaccia a Dio che io procuri la morte di nessun cavaliere», dice.

La sua formazione è ancora in fieri: Curial è ancora fragile. Melchiorre è costretto a dirgli di non comportarsi da femmina, di asciugare le lacrime, che ha troppo facili. Il che non è da cavaliere...

Curial ne ha di strada da fare! Per ora la sua personalità presenta molteplici aspetti, all'interno dei quali deve trovare la sua via. La forza interiore è ben più difficile da raggiungere di quella che Curial mostra di avere nei combattimenti...

Quella di Curial è una formazione complicata. Non si tratta tanto di formare un vincitore di combattimenti, quanto di dargli la struttura interna per andare avanti nel mondo.

Abbiamo detto che per ora Curial non vuole procurare la morte di un qualche cavaliere. Eppure è necessario che Melchiorre vegli su di lui, perché dica di no alla proposta del duca di Baviera: il suo matrimonio con la figlia avrebbe procurato la morte di Guelfa... La morte di un cavaliere no, ma per la morte di una donna, non sembra mostrare la stessa attenzione. E il tema offrirebbe un lungo discorso che potrebbe confermare quanto stiamo dicendo, ma che rimando a un altro luogo, per non complicare ulteriormente il nostro percorso.

Ho già scritto da qualche parte che le questioni formali da sole non riescono a identificare le tradizioni: lo sbandierato primato della forma di certa recente critica ha finito con il fossilizzare i testi, dimenticandone il contenuto. Il contenuto del Curial, affianca le questioni formali per risolvere gli enigmi che il romanzo pone.

Spesso il concetto stesso d'intertestualità viene ormai vastamente frainteso. L'intertestualità non è un richiamo citazionistico di testi ad altri testi, ma un intersecarsi di contesti, di cui ci si accorge tramite precisi segnali testuali. Il procedimento, ciò̀, a un certo punto scompare, a vantaggio di un'illuminazione di fondali che rischierebbero altrimenti di sfuggirci.

Siamo in un momento critico nel quale le polemiche sembrano prolificare e talvolta la vis polemica ci accomuna, il che non è in assoluto sterile, ma anzi estremamente stimolante. Talvolta non essere d'accordo è una sensazione gratificante, se non altro per potersi confrontare su dei fatti e delle opinioni. Di solito si discute sulla legittimità di un metodo e non sui suoi eventuali risultati. Comunque meglio essere un «difensore del dissenso», piuttosto che un «agente dell'Impero», lo ripeto.

Tornando al Curial, mi sembra che sia proprio il contenuto quello che ancora ci può dare delle risposte ai dubbi che le questioni formali hanno messo in evidenza. Chi è Curial? La costruzione del personaggio forse riesce a dirci qualcosa sul nostro autore, a portarci verso la messa a fuoco del suo modo di procedere, di pensare, e chiederci se corrisponde all'identificazione proposta da Soler. In Curial la convivenza dei modelli ci incanala all'interno di quella di chi ha scritto il romanzo. La sua costruzione dei meccanismi è estremamente innovativa. Nel percorso che il genere letterario 
attraversa dal canone medievale a quello moderno, il Curial e Güelfa rappresenta una tappa assai importante: al centro dell'intreccio c'è un personaggio che ha una sua evoluzione interiore: abbiamo visto come questo suo processo fa i suoi primi passi all'inizio del romanzo, potremmo continuare, ma quello che è certo è che Curial passa per delle esperienze che determinano una sua evoluzione che è proprio quella grazie alla quale avremo un lieto fine: Jacob Xalabin è lo stesso dall'inizio alla fine della sua storia, Tirant non so... forse anche, ma Curial mi pare che dei tre è quello che meglio riesce a fare tesoro dell'esperienza, prima guidato da Melchiorre, poi, forse anche da solo, riesce a essere meno vittima di quanto gli capita. Il suo autore ha una visione ottimista degli eventi...

Certo, per dirla con la Meneghetti, «niente separa il romanzo medievale da quello moderno: esiste solo il continuo fluire e trasformarsi di un genere che ha saputo rinnovare nei secoli tutta la sua vitalità».

Comunque, però, questo o quel protagonista dovrà calarsi totalmente all'interno della società in cui verrà posto, che se non sarà quella dell'autore ne sarà senz'altro influenzata: i tempi e i luoghi rappresentati non saranno solo quelli dei personaggi, ma risentiranno di quelli dell'autore. L'identità del romanzo esce dai confini ben definiti della storiografia, esce da un tempo definito e da una geografia reale, per trasformarsi in epoche e siti molteplici, complessi, all'interno dei quali è possibile un'apertura culturale diversa.

Abbiamo visto che Curial non combatte contro chi lo ha accusato, ma contro chi ha accusato di adulterio Cloto, duchessa d'Austria, cugina della regina di Ungheria, e prima ancora contro chi ha accusato un vecchio cavaliere di omicidio, operando quello che oggi in psicologia si chiama spostamento, transfert, cioè un processo automatico e inconscio, attraverso il quale una minaccia interna, derivante da un impulso inaccettabile, viene spostata su un oggetto sostitutivo.

Grazie allo spostamento, al transfert, questa minaccia, che non è stata evitata per mezzo della rimozione, viene ora avvertita e riconosciuta come un pericolo esterno, non più apparentemente connesso allimpulso interno inaccettabile. È ora possibile per l'individuo affrontare il pericolo evitando la situazione, l'oggetto, l'animale, la persona pericolosa. Esempi di spostamento sono le fobie. E così che è possiblile la formazione di Curial. 


\section{Bibliografia}

Badia, L. \& Torró, J. (2015) «Ambient internacional i cultura de cort al Curial e Güelfa: primer suplement a l'edició de Quaderns Crema 2011», in Badia L. \& Casanova E. \& Hauf A. (a cura di), Studia mediaevalia Curt Wittlin dicata $=$ Mediaeval studies in honour Curt Wittlin $=$ Estudis medievals en homenatge a Curt Wittlin, Alacant, Institut Interuniversitari de Filologia Valenciana, pp. 51-66.

Butinyà, J. \& Gros, S. (2013) «[rec. a] Curial e Güelfa (2011): a cura de Lola Badia \& Jaume Torró. Barcelona, Quaderns Crema, 755 p. (Sèrie Gran, 26)», Estudis Romànics, 35, pp. 22-527.

Cambi, F. (a cura di) (2000) Nel Conflitto delle emozioni. Prospettive pedagogiche, Roma, Armando.

Compagna, A. M. (1995) «La storiografia come forma di narrativa complessa: tradizione e innovazione della narrativa catalana», in Beggiato F. \& Cingolani S. (a cura di), La narrativa in Provenza e Catalogna nel XIII e XIV secolo. Atti del Colloquio tenuto a Roma il 12-14 maggio 1993, Pisa, Ets, pp. 217-233.

- (2008-2009) «I risvolti sopranazionali dell’identità catalana: ilcavaliere è turco, è lombardo, è bretone», in Di Girolamo C. \& Di Luca P. \& Scarpati O. (a cura di), La Catalogna in Europa, l'Europa in Catalogna. Transiti, passaggi, traduzioni. Atti del IX Congresso internazionale dell'Associazione italiana di studi catalani (Venezia, 14-16 febbraio 2008) http:/ / www.filmod.unina.it/aisc/attive/.

Ferrando Francés, A. (1997) «Sobre el marc històric de Curial e Güelfa i la possible intencionalitat de la novel la», in Barberà J.-M. (a cura di), Estudis crítics sobre Tirant lo Blanc $i$ el seu context, Barcelona, Centre Aixois de Recherches Hispaniques/Institut Interuniversitari de Filologia Valenciana/Publicacions de l'abadia de Montserrat, pp. 323-408.

_- (ed.) (2012) Estudis lingüistics i culturals sobre Curiale Gü̈lfa, Benjamins, Amsterdam/Philadelphia.

Meneghetti, M. L. (2006) Il romanæo nel Medioevo. Francia, Spagna, Italia, Bologna, Il Mulino.

Soler, A. (2017a) «Enyego d'Àvalos, autor de Curial e Güelfa?», Estudis Romànics, 39, pp. 137-165.

- (2017b) La cort napolitana d'Alfons el Magnànim: el context de Curial e Güelfa, València, PUV, 3 voll.

Thompson, S. (1979 [1 1 $^{\mathrm{a}}$ ed. in inglese 1946]) La fiaba nella tradizione popolare, Milano, Il Saggiatore. 